\title{
Review
}

\section{Medical Principles and Practice}

Med Princ Pract 2013;22:323-333

DOI: $10.1159 / 000346296$
Received: May 21, 2012

Accepted: December 5, 2012

Published online: January 18, 2013

\section{Clinical Errors and Medical Negligence}

\author{
Femi Oyebode \\ University of Birmingham, National Centre for Mental Health, Birmingham, UK
}

\section{Key Words}

Clinical errors · Medical negligence · Medical malpractice .

Patient safety

\begin{abstract}
This paper discusses the definition, nature and origins of clinical errors including their prevention. The relationship between clinical errors and medical negligence is examined as are the characteristics of litigants and events that are the source of litigation. The pattern of malpractice claims in different specialties and settings is examined. Among hospitalized patients worldwide, 3-16\% suffer injury as a result of medical intervention, the most common being the adverse effects of drugs. The frequency of adverse drug effects appears superficially to be higher in intensive care units and emergency departments but once rates have been corrected for volume of patients, comorbidity of conditions and number of drugs prescribed, the difference is not significant. It is concluded that probably no more than 1 in 7 adverse events in medicine result in a malpractice claim and the factors that predict that a patient will resort to litigation include a prior poor relationship with the clinician and the feeling that the patient is not being kept informed. Methods for preventing clinical errors are still in their infancy. The most promising include new technologies such as electronic prescribing systems, diagnostic and clinical decision-making aids and error-resistant systems.
\end{abstract}

Copyright $\odot 2013$ S. Karger AG, Basel

\begin{tabular}{ll}
\hline KARGER & $\begin{array}{l}\text { ( } 2013 \text { S. Karger AG, Basel } \\
1011-7571 / 13 / 0224-0323 \$ 38.00 / 0 \quad \text { Karger }\end{array}$ \\
E-Mail karger@karger.com & $\begin{array}{l}\text { This is an Open Access article licensed under the terms of the } \\
\text { www.karger.com/mpp }\end{array}$ \\
& $\begin{array}{l}\text { Creative Commons Attribution-NonCommercial 3.0 Un- } \\
\text { ported license (CC BY-NC) (www.karger.com/OA-license), } \\
\text { applicable to the online version of the article only. Distribu- } \\
\text { tion permitted for non-commercial purposes only. }\end{array}$
\end{tabular}

\section{Introduction}

Clinical errors and malpractice claims are increasingly important aspects of medical practice. There is concern that the risk of acquiring disabling illness due to medical intervention during hospitalization is contributing to the cost of care, adding to the burden of the patient and as a result of malpractice claims, causing mounting and spiralling costs to the health-care system and for society at large. The Institute of Medicine (IOM) report estimated the total cost of medical error as USD 17-29 billion per annum [1]. The report also estimated that there were 7,000 additional deaths per annum due to medical errors. The aim of this paper is to produce a narrative review of the literature on clinical errors and malpractice claims, respectively, with a view to describing the patterns, costs, consequences and prevention of clinical errors and malpractice claims as well as describing the complex relationship between the two. The literature is extensive but is almost entirely from the USA.

\section{Clinical Errors}

\section{Definition and Patterns of Error}

In the IOM report 'To Err Is Human: Building a Safer Health System' [1], clinical error was defined as 'the failure of a planned action to be completed as intended or the use of a wrong plan to achieve an aim'. In other words,
Dr. Femi Oyebode

University of Birmingham, National Centre for Mental Health

25 Vincent Drive, Edgbaston

Birmingham B15 2FG (UK)

E-Mail Femi.oyebode@ bsmhft.nhs.uk 
errors can arise in planning actions or in executing them. The report listed adverse drug events (ADEs) and improper transfusions, surgical injuries and wrong-site surgery, suicides, restraint-related injuries or death, falls, burns, pressure ulcers and mistaken patient identities as examples of clinical errors. It commented that high error rates with serious consequences are most likely to occur in intensive care units, operating rooms and emergency departments.

It is widely accepted that medication error is the most common and preventable cause of patient injury. This includes the giving of the wrong drug or dose, by the wrong route of administration, to the wrong patient or at a wrong time. There is evidence that in acute hospitals the incidence of ADEs is 6.5/100 admissions and that $1 \%$ of these are fatal, $12 \%$ are life-threatening and $28 \%$ are preventable [2]. Furthermore, it is estimated that there are 5.3 medication errors/100 prescriptions. These include missing dose (53\%), dose errors (15\%), frequency errors $(8 \%)$ and route errors $(5 \%)$, but only $1 \%$ of them were associated with ADEs [2]. A considerable proportion (21\%) of medication errors went undetected by pharmacists during the dispensing process and $23.5 \%$ of the undetected errors were likely to cause ADEs. The majority of overlooked errors were incorrect medication, incorrect strength and incorrect dosage [3]. It is notable that errors occurring during the peri-discharge period in hospitalized patients and those following discharge were most easily missed $[4,5]$. It is also estimated that the additional annual cost of ADEs in hospitalized patients in a 700bed teaching hospital is USD 5.6 million [6]. More recently, the additional cost of ADEs in community hospitals has been estimated as USD 3,000 per patient on average and an increase in length of stay of 3.1 days, similar to that found for academic institutions [7]. In the elderly, ADEs added USD 65,631 to the cost per 1,000 elderly people managed within an ambulatory setting [8]. Perhaps more importantly, many of the injuries caused by medical errors were due to substandard care $[9,10]$ and many were also preventable $[2,11]$.

\section{Drug Error Settings}

The rates of medication errors in pediatric settings appear to be up to three times the rates in adult settings and are reported to be $5.7 \%$ resulting in $0.24 \%$ of ADEs [12]. There are also comparable medication errors in ambulatory pediatric settings where the majority of preventable errors were due to parental drug administration $[13,14]$. The rates in intensive care units do not appear to be different from those in other hospitalized patients, once the number of ordered and administered drugs had been controlled for [15]. The rates in psychiatric hospitals are similar to those in other settings [16]. In nursing homes for the elderly, there were $1.89 / 100$ resident-months of ADEs and 0.65/100 resident-months of potential ADEs [17]. In this population, of the ADEs, $6 \%$ were life-threatening and $38 \%$ were serious. In total, $51 \%$ of the ADEs were judged to be preventable. ADEs in nursing homes commonly involved the use of psychotropic agents resulting in neuropsychiatric side effects, falls and oversedation. In ambulatory elderly people, the rate of ADEs was 50.1/1,000 person-years. Many of these ADEs were serious, life-threatening or fatal (38\%) and it was judged that $42 \%$ were preventable [18]. The most commonly implicated drugs were cardiovascular agents, diuretics, nonopioid analgesics, hypoglycemic drugs and anticoagulants. The risk of ADEs was markedly elevated in elderly patients who had multiple conditions, were receiving multiple drugs including nonopioid analgesics, anticoagulants, diuretics and anticonvulsants [19]. ADEs in ambulatory settings such as primary care are common [20] and also occur in ambulatory adult and pediatric chemotherapy settings [21].

A systematic review of 22 studies involving 3,755 patients found that errors in prescription medication histories occurred in up to $67 \%$ of cases and these included commission errors as well as omissions, incorrect dose and incorrect frequency. For example, a patient who was admitted in a coma had her medication brought in by relatives including propafenone that had been discontinued months before [22].

In summary, prescription errors associated with drug errors and ADEs were frequent in practically all healthcare settings. However, it is difficult to make ready comparisons because rates are reported differently across studies. Some studies report rates per 100 admissions, others report rates per 100 resident months, whilst others report rates per 1,000 person-years. This is a fundamental problem that needs to be resolved.

\section{Nondrug Events}

In addition to ADEs, other adverse events in hospitalized patients include wound infections and technical complications. It is estimated that nearly half of all adverse events in hospitals are associated with surgical operations. Those adverse events not associated with surgery included diagnostic mishaps, therapeutic mishaps (errors of omission) and events occurring in accident and emergency departments [11]. Diagnostic errors rather than technical mishaps or adverse consequences of med- 
ication errors are also relatively common. For example, delay or diagnostic errors in cervical spine injuries occurred in approximately $4 \%$ of cases; this points to the need for the appropriate use of a standard 3-view cervical spine series with careful interpretation of the images [23].

\section{Global Picture}

Much of the work on clinical and medication errors is from the USA. However, in a recently reported study from a typical British teaching hospital over a 4-week study period, $1.5 \%$ prescribing errors were identified and potentially serious errors occurred in $0.4 \%$ of cases. The majority of errors originated from prescribing decisions [24]. In general practice, Rubin et al. [25] developed a classification system for errors and reported an overall error rate of 75.6/1,000 appointments. The majority of errors were prescription errors (42\%) followed by communication errors (30\%).

The relative paucity of data on patient safety in countries other than the USA and in particular in developing and emerging economies has been commented upon [26, 27]. There have been calls for knowledge sharing, arguing for the development of an international knowledge database [28]. There are increasing numbers of reports on clinical errors from other countries [29-34]. The evidence suggests that $3-16 \%$ of hospitalized patients suffer harm as a result of medical care, which indicates the considerable burden of harm to patients [26].

\section{Explanatory Models of Human Error}

There are two models of causation of human error, namely the person approach and the systems approach. The person approach focuses on the errors of individuals, and tends to blame individuals for forgetfulness, inattention or moral failure. The systems approach identifies the conditions and systems under which individuals work as the source of the error with the aim of both understanding the origin of errors and building defences to avert them or mitigate their effects. An important weakness of the person approach is that it isolates unsafe acts(s) from the systems context [35]. Nonetheless, human rather than technical failures underlie much of the errors that are identified and, in general, represent the greatest threat to potentially hazardous systems [36].

The most common systems failures identified as underlying clinical errors are failures in dissemination of drug knowledge and inadequate availability of patient information such as test results necessary for safe treatment. In all, 7 systems failures account for $78 \%$ of the identified errors [37]. There is though, a hidden aspect to systems failures. In a study of 3 pediatric cardiac teams, the respondents were well aware of the burden of potential errors and reported that errors occurred repeatedly and that guidelines and policies were often disregarded. In addition, the respondents identified staffing levels, unavailability of equipment, production pressure and hectic schedules as matters for concern, but felt unable to either express their concerns or disagreement [38]. In other words, despite the respondents being aware of rules and regulations, these were often disregarded. This raises questions about individual motivation, professional culture and social constraints within the workplace. Aside from workplace factors such as those described above, fatigue that derives from night work and schedule instability has been reported to be associated with clinical errors in $42 \%$ of a group of junior doctors anonymously surveyed in a New Zealand study [39]. Frequent shift work of $24 \mathrm{~h}$ or more was more likely to be associated with medical errors than shorter shifts [40]. In a study of nurses, it has also been shown that interruptions during the preparation and administration of drugs were associated with an increased likelihood of procedural and clinical errors and the frequency of interruptions was highly correlated with the more severe errors [41]. Furthermore, the nurse-to-patient ratio has been found to be significantly positively associated with the increased likelihood of adverse events. This was true for an urban teaching hospital that had greater than $100 \%$ occupancy rates for much of a year. An increase in nurse-to-patient ratio of 0.1 was associated with a $28 \%$ increase in adverse events [42]. In the USA, teaching hospitals had higher ADEs than rural hospitals, but the rates of ADEs due to negligence were lower in teaching hospitals and for-profit institutions compared with hospitals that cared predominantly for minority groups [9].

However, despite the importance of the systems context, an understanding of the personal and motivational dimensions to clinical errors also remains important. In a study of general practitioners (GPs) in Spain, an adverse event rate of 10.6/annum was reported for each doctor. Most of these were drug side effects (37\%) and diagnostic delays $(33 \%)$ [43]. What is surprising though, was that $28 \%$ of the doctors claimed that they never made mistakes, a group the researchers termed 'deniers'. This attitude to there being a potential for error in all human contexts is hardly reassuring. Other factors that have been reported include slips in attention, not applying prescribing rules, the low perceived importance of prescribing, the hierarchical nature of medicine and the absence of self-awareness of error [24]. There is also evidence that 
cognitive factors involving illness, medications, stress, alcohol and emotional disturbance can be involved in medical errors [44].

There is little doubt that the person approach continues to hold sway and that the temptation to blame an identified individual is hardly resisted by institutions. Nonetheless, there is much that can be done to improve the work environment so as to reduce the likelihood of clinical error. Some of the tactics that have been suggested are: (1) to reduce the complexity of tasks, (2) to optimize information processing by the use of protocols or aids, (3) to automate wisely and as necessary, (4) to use constraints such as is the case with diesel and unleaded petrol dispensers and (5) to mitigate the unwanted side effects of change particularly when new techniques or treatments are first introduced. Additionally, protocols that improve communication between clinicians have been identified by inquiries into homicides by psychiatric patients as likely to reduce adverse outcomes [45]. In summary, clinical errors arise within a system. Although individual factors are important, any overemphasis on the personal aspects of errors is unlikely to provide a basis for developing error-resistant systems.

\section{Prevention}

There are numerous proposed strategies for reducing the incidence of clinical errors. The IOM [1] report, and the response of the US government to it [46], advocated establishing a national focus in order to create leadership and research tools to enhance the knowledge base about patient safety. It also advocated a nationwide public mandatory reporting system that would help to identify and learn from errors including the desire to ensure that rootcause analysis not be discoverable during malpractice litigation. Furthermore, it called for improvement in safety through the actions of oversight organizations, professional groups and others. Finally, it directly asked healthcare organizations to ensure safe practices at the delivery level.

In the UK, the Chief Medical Officer's report 'An Organisation With A Memory' [47] called for changes in the NHS which should include unified mechanisms for reporting and analysis when things go wrong, a more open culture in which errors or service failures can be reported and discussed, mechanisms for ensuring that where lessons are identified the necessary changes are put into practice and a much wider appreciation of the value of the system approach in preventing, analyzing and learning from errors. There is certainly a convergence of approach and thinking across the Atlantic. However, this conver- gence of views about the benefits of reporting are not shared across the board by clinical teams or health-care organizations [48] and there is little good evidence that error identification systems are of much use in teaching residents, for example $[49,50]$, or indeed in reducing error rates. Nonetheless, calls for incident reporting continue $[51,52]$. Root-cause analysis was borrowed from the defense industry by health-care organizations and promulgated as a means of reducing risk and improving patient safety, but doubts abound about its potential to achieve this $[53,54]$.

At a more practical level, the rhetoric calling for a move from perceiving medical errors as deriving from individual failure to systems failure and from undue reliance on independent, individual performance excellence to a culture of interdependent, collaborative, interprofessional teamwork [55] has resulted in more team training in order to improve patient safety $[56,57]$ and has demonstrated an improvement in patient safety as a result of such training [58]. A special aspect of this is training specifically for emergencies using approaches derived from the airline industry [59]. A more extensive team-training exercise was the collaboration across hospitals to alter processes and thereby embed patient safety into hospital culture. It is clear from the report of that collaboration that strong leadership is required to secure change, even recalcitrant issues such as nonpunitive reporting can become institutionalized [60] and that once momentum is established, it is possible to continue to make stepwise progress [61].

The use of voice-recognition technology for radiology reports and the manifest error rates have been studied as a means of drawing attention to the risk attendant on the use of new technologies [62]. The use of electronic prescribing and information technology systems has been extensively investigated as a method for reducing prescribing errors $[2,21,63-65]$ and has been shown to be effective [66-68]. The participation of a pharmacist (as a member of the clinical team) on clinical rounds also appears to reduce the likelihood of ADEs $[69,70]$. The use of computer-based protocol reminders appears to reduce errors, irrespective of the seniority of the clinician [71], and is advocated as a means of improving patient safety $[72,73]$. There do not appear to be any patient factors that significantly predict the likelihood of ADEs [67], so clinical characteristics may not be an easy means of determining such a likelihood. Computer-assisted decision-making also appears to be helpful [74], as are systems for aiding diagnostic accuracy using algorithms, checklists, instructions and guidelines [75]. Other technologies that 
may be useful in reducing medical errors are bar coding, smart pumps and computerized error monitoring [76].

It is argued by some commentators that the desire to reduce medical errors by using a combination of incident reporting and emphasizing a systems approach is not likely to be helpful since the behaviors of clinicians are predicated on personal identities that may be opposed to guidelines and/or protocols [77]. Furthermore, professional independence, suspicion of managers and the values of clinicians may be significant obstacles to a systems approach for reducing clinical errors [78]. In summary, a systematic approach to the development of methods to prevent clinical errors is in its infancy. There is an absence of a consistent, widely accepted and demonstrably effective method. The adoption of new technologies to aid diagnosis and clinical decision-making and the introduction of electronic prescribing systems appear to be the most promising.

\section{Medical Negligence}

Medical negligence claims are not coextensive with cases that are the subject of a clinical error. The Harvard Medical Practice study reported that the overall rate of negligence claims per discharge was $0.13 \%$ [79]. Of the 280 patients in the study who had experienced adverse events caused by clinical error, only 8 filed a medical malpractice claim. This gave an estimate of the ratio of adverse event to malpractice claim of 7.6:1. The authors concluded that this was a clear overestimation, as most of the events for which malpractice claims were made did not meet the research criteria of adverse events due to clinical error. In the UK, it was estimated that there were 90,000 adverse events per annum, of which 13,500 involved the death of patients, but only resulted in around 7,000 claims and 2,000 payments [80].

In 1996/1997, medical negligence was estimated to cost the National Health Service (NHS) in England GBP 235 million, and in 2010/2011 this figure had risen to GBP 863 million [81]. Negligence claims against GPs went up 13fold between 1989 and 1998. For example, it was reported that in 1989 there were 38 claims against GP members of the Medical Protection Society, and by 1998 these claims had risen to 500. The greatest rise was in the number of actions that ultimately failed or were abandoned [82]. However, it must be noted that unlike data from medical protection organizations, estimates of negligence claims in the NHS are subject to revision. Some even argue that such estimates are exaggerated and that although the rate

Clinical Errors and Medical Negligence of growth has increased, this does not amount to an uncontrollable explosion as is sometimes inferred [83]. The rising number of malpractice claims reported in the UK is mirrored in other jurisdictions. In the period 1999-2008, in Saudi Arabia, claims rose from 440 to 1,356. Most claims were in obstetrics followed by general surgery [84].

\section{Patterns and Determinants of Malpractice Claims}

In another study, ADEs represented $6.3 \%$ of malpractice claims and $73 \%$ of these were judged to be preventable; half of the errors occurred in outpatients and most frequently involved antibiotics, antidepressants, antipsychotics, cardiovascular agents and anticoagulants [85]. A substantial proportion of ADEs (13\%) resulted in litigation at a cost of USD 3.1 million [86]. A specific example of an ADE resulting in a malpractice claim is the use of corticosteroids. Allegation of negligent use was the most common reason for a claim (65\%), followed by lack of proper informed consent (36\%), and failure to diagnose or misdiagnosis (22\%) [87].

The pattern and determinants of litigation vary across specialties and the literature is unsystematic. The following survey is for illustrative purposes only. Surgical practice attracts a high rate of claims. Malpractice claims following thyroid surgery often involve laryngeal nerve damage (45\%). However, there is little evidence that laryngeal nerve monitoring has influenced the pattern of claims in the 20-year period 1989-2009 [88, 89]. Over the 22 -year period 1985-2007, urology ranked 12th of 28 specialties in the number of claims reported. Improper performance and diagnostic errors constituted $51 \%$ of the claims, often involving malignancies of the prostate, testis and kidneys [90]. Specifically, missed diagnosis constituted $15 \%$ of claims in another study, and $71 \%$ of urological missed diagnosis were cancers, with an average of USD 526,460 of damages being paid out. This is disproportionately greater than damages paid out for other missed diagnosis claims [91]. In bariatric surgery, an analysis of 100 malpractice claims found that the mean age of patients was 40 years and $75 \%$ were females with $81 \%$ having a body mass index $>60$. One-third of the cases were diabetic and $38 \%$ had sleep apnea. In summary, these were severely vulnerable patients, yet only $22 \%$ had completed detailed consent forms. The surgeons had been practising for over 1 year (42\%) and $26 \%$ had treated over 100 cases each. Intraoperative complications occurred in $32 \%$. Adverse events included leaks (53\%), intra-abdominal abscess (33\%), bowel obstruction (18\%) and major airways events (10\%). There were 53 deaths and $7 \%$ had major disabilities [92]. 
The vast majority of malpractice claims in ear, nose, throat, head and neck surgery were for the nose and sinuses (34\%) and involved mostly improper performance in $50 \%$ of the cases [93]. The most common complications of endoscopic sinus surgery resulting in malpractice claims included cerebrospinal fluid leak, orbital trauma and anosmia [94]. In otology, in a study investigating claims over a 15 -year period, there were 137 claims, representing $26 \%$ of all the claims in otolaryngology. Of the 97 successful claims, 63 were related to operative complications. This included 6 cases of wrongside/wrong-site surgery, and 15 cases of inadequate informed consent. The most common injuries were hearing loss, facial paralysis and additional/unnecessary surgery [95].

Orthopedic adult reconstruction subspecialists are sued for alleged medical malpractice at a rate of over twice that of the physician population as a whole, and the rate appears disproportionately high in the first decade of practice. The overall risk of a malpractice claim is related to the number of years spent in practice. After 30 years in an adult reconstruction practice, the cumulative rate of being sued at least once is over $90 \%$. The degree to which the likelihood of a suit occurs is thought to be related to factors such as practice setting and size, fellowship training, years in practice, volume and location of practice, but this has yet to be firmly demonstrated [96]. Following hip/knee replacement surgery, nerve injury was the most commonly cited source of litigation, followed by limblength discrepancy, infection, vascular injury, hip dislocation, compartment syndrome, deep vein thrombosis, chronic pain and periprosthetic fracture [97].

Surprisingly, trauma care has one of the lowest malpractice claim rates in medicine [98].

In ophthalmology, malpractice claims in the USA are infrequent. In the UK between 1995 and 2006, there were 848 claims with a total cost of GBP 11 million. Cataracts were most likely to result in claims and the highest mean damages paid out were for pediatric ophthalmology, with claims related to glaucoma being the most likely to result in damages (GBP 170,000) [99].

In Tehran, a study of malpractice claims in dentistry revealed that most claims arose in the context of fixed prosthodontics and oral surgery in private practice [100].

Like the other surgical specialties, obstetrics and gynecology attract significant malpractice claims. A survey of fellows of the American College of Obstetricians and Gynecologists in 2006 found that $89 \%$ had been sued during their careers, and $37 \%$ had been sued once during their residency period. There were 2.6 claims per individual and $62 \%$ of claims were for obstetric practice rather than for gynecology [101].

Cerebral palsy is associated with some of the largest damages paid out in many jurisdictions. In the UK, in the period 1984-1993, 20\% of cerebral palsy births resulted in malpractice claims. The presence of the 3 essential template criteria for determining acute intrapartum hypoxia did not appear to influence the outcome of the cases [102]. A Swedish study on severe fetal asphyxiation due to malpractice between 1990 and 2005 reported that the most common errors were failure to supervise fetal wellbeing (98\%), neglect of signs of fetal asphyxiation (71\%), incautious use of oxytocin (71\%) and choosing a nonoptimal mode of delivery (52\%) [103]. In Japan, cerebral palsy accounted for $50 \%$ of birth-related claims [104].

Investigative specialties also have their share of malpractice claims. In radiology, the incidence of malpractice claims is estimated to be 44/1,000 events. Missed breast abnormalities were the most common followed by missed bone abnormalities [105]. Complications of radiological interventions occurred in $10 \%$ of claims and there were a few claims resulting from failure to order further diagnostic examinations $[105,106]$. Another study showed that one-third of Italian radiologists had been sued and there was evidence of significant associated stress and work dissatisfaction resulting from the experience [107]. The median delay in diagnosis of primary breast cancer in a Swedish study was 11 months and in recurrent breast cancer was 3.5 months. The delay was thought to have an adverse impact on therapy in $23 \%$ of cases and an adverse effect on outcome in $11 \%$. The delays were mainly caused by incomplete clinical or radiological examinations [108].

Gastrointestinal endoscopy can result in malpractice claims. The reported claims included esophagoduodenoscopy (44\%), colonoscopy (22\%), endoscopic sphincterotomy (22\%) and endoscopic retrograde cholangiopancreatography (11\%). Most of the claims alleged performance error or lack of informed consent [109].

In a study of the medicolegal aspects of malpractice claims in pathology, 171 legal cases were identified from 1988 to 2005 . Nearly half of these involved surgical pathology; among the remainder, cytology cases slightly outnumbered those pertaining to clinical pathology. Among the surgical pathology cases and overall, the most common reason for a medical malpractice lawsuit relating to pathology was the alleged missed diagnosis of melanoma on a skin biopsy specimen; less commonly, they involved breast biopsy and gynecological specimens. Among the 48 cases related to cytology, 37 involved falsenegative cervical smears. Less common were cases related 
to fine-needle aspirates of the breast or thyroid or cytology specimens of the lung. Among the 36 cases involving clinical pathology, 32 related to the blood bank, usually transfusion-acquired human immunodeficiency virus infection [110].

The role of poor communication in determining the likelihood of malpractice litigation is poorly understood. In Japan, there is evidence that the courts are starting to regard inadequate information-giving to patients as a breach of duty of care [111]. But, perhaps more importantly, the doctor's explanations and communication with patients appear to influence the likelihood of malpractice claims $[112,113]$.

It is evident from the above examples that the relationship between clinical errors and malpractice claims is complex. Certainly, not all clinical errors result in malpractice claims and not all malpractice claims are the result of clinical errors. In one particular study, in 3\% of the claims, there were no verifiable medical injuries, and 37\% did not involve errors [114]. Another documented the factors that influence patients' decisions to make a claim: (1) a poor relationship with providers or clinicians before the alleged injury, (2) television advertising by law firms, (3) explicit recommendations by health providers or professionals to seek legal advice, (4) having the impression of not being kept informed and (5) financial concerns [115]. This was an American study and may not be directly applicable to other countries. This limitation is particularly relevant to the financial concerns underlying the progression of a clinical error to a negligence claim, as this seemed to become prominent in those in employment when the outstanding medical bills equalled or exceeded $50 \%$ of their annual income. The ADEs most likely to result in negligence claims involve the use of antibiotics, antidepressants or antipsychotic drugs, cardiovascular drugs and anticoagulants. These events are often severe, costly and preventable and about half occur in outpatients.

\section{Criminalization of Clinical Errors}

The most worrying aspect of the recent changes in public attitude to clinical error is the growth in the criminalization of fatal medical errors. Ferner [116] reported on the dramatic increase in the use of the charge of manslaughter in the UK against doctors during the period 1970-1999. In the periods 1970-1979 and 1980-1989, there were only 2 cases each whereas in the period 1990 1999, there were 13 cases involving 17 doctors [117]. Ferner concluded 'For most errors ... the criminal law is unsatisfactory. Convicting doctors of manslaughter may satisfy a desire for retribution, but deters careful consideration of the ways of preventing tragedies from recurring'. This increase is attributed to society's changed attitude towards the notion of gross negligence. For example, in 1925, the Court of Appeal stressed the importance of the negligence having to be gross when it said the accused's negligence must go beyond a mere matter of compensation between subjects and show such disregard for the life and safety of others as to amount to a crime against the state and conduct deserving of punishment. In a 19th century case, the court noted that if there was only the kind of forgetfulness which is common to everybody, or if there was a slight want of skill... it would be wrong to proceed against a man criminally in respect of such injury'. The court then gave as an example of gross negligence the surgeon who operated while drunk [118]. These changes are paralleled by the public's view that doctors ought to be charged with manslaughter for fatal clinical errors [119]. Italy has the highest rate of criminal proceedings against doctors in the context of clinical errors [120]. It is also worth noting that Taiwan stands out as a jurisdiction where medical errors are treated as criminal offences as a matter of routine. One Taiwanese physician is found guilty every 3 months; curiously, the grounds for the disputes are a delayed or missed diagnosis and surgical complications indistinguishable from those in other parts of the world [121].

\section{Effects of Malpractice Claims}

It is arguable whether or not negligence claims and the increasing damages paid out serve to deter further clinical error. Although medical practitioners may alter their practice such that it appears to be more legally defensible, there does not appear to be an accompanying decrease either in claims or errors. Furthermore, the idea that health-care providers would participate in quality improvement schemes and clinical error-reduction initiatives because of a desire to curb the spiraling costs of negligence claims is not borne out by the facts [122]. This is partly due to the misfit between who is injured by medical negligence and who sues, but also by the externalization of the costs of negligence to third parties. Furthermore, defense attorney costs account for a considerable proportion of defense costs whether or not the cases go to trial; in one study this was 74\% [123]. The incentive for healthcare providers to act to reduce clinical errors may not be particularly strong. However, even if it were true that health care acted vigorously to reduce the likelihood of clinical errors by, for example, adopting and insisting on the use of evidence-based guidance as part of clinical gov- 
ernance or a quality improvement scheme, there are questions about how far this would in fact influence the incidence of medical negligence.

One of the undesirable consequences of medical malpractice claims is defensive medicine, which can be defined as the modification of clinical practice solely to reduce exposure to legal challenges by patients rather than for direct clinical reasons [124]. A survey of Italian GPs and specialists found that $37 \%$ of GPs and $83 \%$ of specialists (comprising surgeons and anesthetists) had engaged in at least one defensive practice in the previous month [124]. It is arguable how far defensive medicine contributes to the increasing costs of medical care. In orthopedics, there is good evidence that defensive practice is associated with prior experience of a malpractice claim. In an American study, defensive imaging represented 19.1\% of imaging and $34.7 \%$ of the total cost. Magnetic resonance imaging (MRI) represented $48.7 \%$ of the defensive imaging, and $38.5 \%$ of 501 MRIs were ordered for defensive reasons. The proportion of defensive imaging ordered by orthopedic surgeons who had been sued for medical malpractice within the previous 5 years was significantly greater than the proportion ordered by those who had not been sued within the same time frame [125]. Nonetheless, there is as yet no conclusive evidence that defensive medicine adds substantial costs to health care. There is some consensus that the influence is, at most, marginal [126]. It is important to emphasize that aside from the additional costs of defensive medicine, some of the unnecessary costs of health care can be attributed to the motivation to earn supplementary incomes by administering unnecessary tests and investigations.

\section{Clinical Guidelines and Malpractice Claims}

Clinical guidelines have been systematically developed to assist clinical decision-making. In medical malpractice claims and in court, these guidelines may act as a source of information, provided they are the product of a recognized body and are deemed reliable. They can be seen as normative standards and used as explicit standards of care at the time of the index clinical event and also to assess the degree to which a questionable practice was in line with accepted standards [127]. Hurwitz [128] argues that if guidelines were to be consulted by courts because they provide evidence of standards justified in relation to evidence rather than custom, this would strengthen what he refers to as the normative dynamic of the law, with the focus shifting from what it is customary to do to what ought to be done; there being the risk, as Hurwitz sees it, of a slavish adherence to clinical guidelines.

\section{Conclusions}

This paper has discussed the increasing public awareness of clinical errors, their extent and their consequences, particularly those that result in ADEs, and the nature and pattern of these errors. There is an overlap between clinical errors and medical negligence claims. Probably no more than 1 in 7 adverse events in medicine result in a negligence claim. It is important to recognize that many negligence claims would not normally be regarded by medical practitioners as arising from adverse events. Nonetheless, the factors that predict that a patient will resort to litigation include a prior poor relationship with the clinician and the feeling that the patient is not being kept informed.

\section{References}

1 Kohn LT, Corrigan JM, Donaldson MS: To Err Is Human: Building a Safer Health System. Institute of Medicine, Committee on Quality of Health Care in America. Washington, National Academy Press, 2000.

-2 Bates DW, Boyle DL, Van der Vliet MB, et al: Relationship between medication errors and adverse drug events. J Gen Intern Med 1995; $10 ; 199-205$

3 Cina JL, Gandhi TK, Churchill W, et al: How many hospital pharmacy medication dispensing errors go undetected? Jt Comm J Qual Patient Saf 2006;32:73-80.
4 Forster AJ, Murff HJ, Peterson JF, et al: The incidence and severity of adverse events affecting patients after discharge from the hospital. Ann Intern Med 2003;138:161-167.

$\rightarrow 5$ Forster AJ, Murff HJ, Peterson JF, et al: Adverse drug events occurring following hospital discharge. J Gen Intern Med 2005;20:317-323.

-6 Bates DW, Spell N, Cullen DJ, et al: The costs of adverse drug events in hospitalized patients. Adverse Drug Events Prevention Study Group. JAMA 1997;277:307-311.

7 Hug BL, Keohane C, Seger DL, et al: The costs of adverse drug events in community hospitals. Jt Comm J Qual Patient Saf 2012;38:120126.
$>8$ Field TS, Gilman BH, Subramanian S, et al The costs associated with adverse drug events among older adults in the ambulatory setting. Med Care 2005;43:1171-1176.

-9 Brennan TA, Hebert LE, Laird NM, et al: Hospital characteristics associated with adverse events and substandard care. JAMA 1991;265: 3265-3269.

10 Brennan TA, Leape LL, Laird NM, et al: Incidence of adverse events and negligence in hospitalized patients: results of the Harvard Medical Practice Study I. 1991. Qual Saf Health Care 2004;13:145-151;disc 151-152. 
11 Leape LL, Brennan TA, Laird N, et al: The nature of adverse events in hospitalized patients. Results of the Harvard Medical Practice Study II. N Engl J Med 1991;324:377-384.

12 Kaushal R, Bates DW, Landrigan C, et al: Medication errors and adverse drug events in pediatric inpatients. JAMA 2001;285:21142120.

13 Kaushal R, Bates DW, Abramson EL, et al: Unit-based clinical pharmacists' prevention of serious medication errors in pediatric inpatients. Am J Health Syst Pharm 2008;65:12541260.

14 Kaushal R, Goldmann DA, Keohane CA, et al: Medication errors in paediatric outpatients. Qual Saf Health Care 2010;19:e30.

15 Cullen DJ, Sweitzer BJ, Bates DW, et al: Preventable adverse drug events in hospitalized patients: a comparative study of intensive care and general care units. Crit Care Med 1997; 25:1289-1297.

16 Rothschild JM, Mann K, Keohane CA, et al: Medication safety in a psychiatric hospital. Gen Hosp Psychiatry 2007;29:156-162.

17 Gurwitz JH, Field TS, Avorn J, et al: Incidence and preventability of adverse drug events in nursing homes. Am J Med 2000;109:87-94.

18 Gurwitz JH, Field TS, Harrold LR, et al: Incidence and preventability of adverse drug events among older persons in the ambulatory setting. JAMA 2003;289:1107-1116.

19 Field TS, Gurwitz JH, Harrold LR, et al: Risk factors for adverse drug events among older adults in the ambulatory setting. J Am Geriatr Soc 2004;52:1349-1354.

20 Gandhi TK, Weingart SN, Borus J, et al: Adverse drug events in ambulatory care. $\mathrm{N}$ Engl J Med 2003;348:1556-1564.

-21 Gandhi TK, Weingart SN, Seger AC, et al: Outpatient prescribing errors and the impact of computerized prescribing. J Gen Intern Med 2005;20:837-841.

22 Tam VC, Knowles SR, Cornish PL, et al: Frequency, type and clinical importance of medication history errors at admission to hospital: a systematic review. CMAJ 2005;173:510515.

23 Davis JW, Phreaner DL, Hoyt DB, et al: The 3 etiology of missed cervical spine injuries. J Trauma 1993;34:342-346.

24 Dean B, Schachter M, Vincent C, et al: Prescribing errors in hospital inpatients: their incidence and clinical significance. Qual Saf Health Care 2002;11:340-344.

25 Rubin G, George A, Chinn DJ, et al: Errors in general practice: development of an error classification and pilot study of a method for detecting errors. Qual Saf Health Care 2003, 12:443-447.

26 Jha AK, Prasopa-Plaizier N, Larizgoitia I, Bates DW, Research Priority Setting Working Group of the WHO World Alliance for Patient Safety: Patient safety research: an overview of the global evidence. Qual Saf Health Care 2010;19:42-47.
27 Carpenter KB, Duevel MA, Lee PW, et al: Measures of patient safety in developing and emerging countries: a review of the literature. Qual Saf Health Care 2010;19:48-54.

28 Cresswell KM, Bates DW, Phansalkar S, et al: Opportunities and challenges in creating an international centralised knowledge base for clinical decision support systems in ePrescribing. BMJ Qual Saf 2011;20:625-630.

29 Famularo G, Salvini P, Terranova A, et al: Clinical errors in emergency medicine: experience at the emergency department of an Italian teaching hospital. Acad Emerg Med 2000; $7: 1278-1281$

30 Sangtawesin V, Kanjanapattanakul W, Srisan P, et al: Medication errors at Queen Sirikit National Institute of Child Health. J Med Assoc Thai 2003;86(suppl 3):S570-S575.

31 Morimoto T, Sakuma M, Matsui K, Kuramoto N, Toshiro J, Murakami J, et al: Incidence of adverse drug events and medication errors in Japan: the JADE study. J Gen Intern Med 2011;26:148-153.

32 Jylhä V, Saranto K, Bates DW: Preventable adverse drug events and their causes and contributing factors: the analysis of register data. Int J Qual Health Care 2011;23:187-197.

33 Sakuma M, Morimoto T, Matsui K, et al: Epidemiology of potentially inappropriate medication use in elderly patients in Japanese acute care hospitals. Pharmacoepidemiol Drug Saf 2011:20:386-392.

34 Madea B: Medico-legal autopsies as a source of information to improve patient safety. Leg Med (Tokyo) 2009;11(suppl 1):S76-S79.

35 Reason J: Human error: models and management. BMJ 2000;320:768-770.

36 Reason J: Understanding adverse events: human factors. Qual Health Care 1995;4:80-89.

37 Leape LL, Bates DW, Cullen DJ, et al: Systems analysis of adverse drug events. ADE Prevention Study Group. JAMA 1995;274:35-43.

38 Bognár A, Barach P, Johnson JK, et al: Errors and the burden of errors: attitudes, perceptions, and the culture of safety in pediatric cardiac surgical teams. Ann Thorac Surg 2008;85:1374-1381.

39 Gander P, Purnell H, Garden A, et al: Work patterns and fatigue-related risk among junior doctors. Occup Environ Med 2007;64: 733-738.

40 Landrigan CP, Rothschild JM, Cronin JW, et al: Effect of reducing interns' work hours on serious medical errors in intensive care units. N Engl J Med 2004;351:1838-1848.

41 Westbrook JI, Woods A, Rob MI, et al: Association of interruptions with an increased risk and severity of medication administration errors. Arch Intern Med 2010;170:683-690.

42 Weissman JS, Rothschild JM, Bendavid E, et al: Hospital workload and adverse events. Med Care 2007;45:448-455

43 Borrell-Carrió F, Páez Regadera C, Suñol Sala $\mathrm{R}$, et al: Clinical error and adverse events: Primary care doctors perception. Aten Primaria 2006;38:25-32.
44 Stripe SC, Best LG, Cole-Harding S, et al: Aviation model cognitive risk factors applied to medical malpractice cases. J Am Board Fam Med 2006; 19:627-632.

45 McGrath M, Oyebode F: Qualitative analysis of recommendations in 79 inquiries after homicide committed by persons with mental illness. J Mental Health L 2002:262.

46 Quality Interagency Coordination Task Force Doing What Counts for Patient Safety: Federal Actions to Reduce Medical Errors and Their Impact. Washington, US Dept of Health and Human Services, Agency for Healthcare Research and Quality, 2000.

47 Milburn A: An organization with a memory: report of an expert group on learning from adverse events in the NHS. London, Department of Health, 2000.

48 Hobgood C, Xie J, Weiner B, et al: Error identification, disclosure, and reporting: practice patterns of three emergency medicine provider types. Acad Emerg Med 2004; 11:196-199.

49 Cullen DJ, Bates DW, Small SD, et al: The incident reporting system does not detect adverse drug events: a problem for quality improvement. Jt Comm J Qual Improv 1995;21: 541-548.

50 Hobgood CD, Ma OJ, Swart GL: Emergency medicine resident errors: identification and educational utilization. Acad Emerg Med 2000; 7:1317-1320.

51 Leape LL: A systems analysis approach to medical error. J Eval Clin Pract 1997;3:213222

52 Kelly SP, Astbury NJ: Patient safety in cataract surgery. Eye 2006;20:275-282.

53 Iedema RA, Jorm C, Long D, et al: Turning the medical gaze in upon itself: root cause analysis and the investigation of clinical error. Soc Sci Med 2006;62:1605-1615.

54 Iedema R, Jorm C, Braithwaite J: Managing the scope and impact of root cause analysis recommendations. J Health Organ Manag 2008;22:569-585.

55 Leape LL: Errors in medicine. Clin Chim Acta 2009;404:2-5.

56 Barrett J, Gifford C, Morey J, et al: Enhancing patient safety through teamwork training. J Healthc Risk Manag 2001;21:57-65.

57 Kilner E, Sheppard LA: The role of teamwork and communication in the emergency department: a systematic review. Int Emerg Nurs 2010;18:127-137.

58 Morey JC, Simon R, Jay GD, et al: Error reduction and performance improvement in the emergency department through formal teamwork training: evaluation results of the MedTeams project. Health Serv Res 2002;37: 1553-1581

59 Schmidt CE, Hardt F, Möller J, et al: Improvement of team competence in the operating room: training programs from aviation. Anaesthesist 2010;59:717-722, 724-726. 
60 Leape LL, Kabcenell AI, Gandhi TK, et al: Reducing adverse drug events: lessons from a breakthrough series collaborative. Jt Comm J Qual Improv 2000;26:321-331.

61 Leape LL, Rogers G, Hanna D, et al: Developing and implementing new safe practices: voluntary adoption through statewide collaboratives. Qual Saf Health Care 2006;15:289-295.

62 Chang CA, Strahan R, Jolley D: Non-clinical errors using voice recognition dictation software for radiology reports: a retrospective audit. J Digit Imaging 2011;24:724-728.

63 Bates DW, Cohen M, Leape LL, et al: Reducing the frequency of errors in medicine using information technology. J Am Med Inform Assoc 2001;8:299-308.

64 Devine EB, Wilson-Norton JL, Lawless NM, et al: Characterization of prescribing errors in an internal medicine clinic. Am J Health Syst Pharm 2007;64:1062-1070.

65 Gawande AA, Bates DW: The use of information technology in improving medical performance. Part III. Patient-support tools. Med Gen Med 2000;2:E12.

-66 Bates DW, Leape LL, Cullen DJ, et al: Effect of computerized physician order entry and a team intervention on prevention of serious medication errors. JAMA 1998;280:13111316.

67 Bates DW, Miller EB, Cullen DJ, et al: Patient risk factors for adverse drug events in hospitalized patients. ADE prevention study group. Arch Intern Med 1999;159:2553-2560.

68 Westbrook JI, Reckmann M, Li L, et al: Effects of two commercial electronic prescribing systems on prescribing error rates in hospital inpatients: a before and after study. PLoS Med 2012;9:e1001164.

69 Leape LL, Cullen DJ, Clapp MD, et al: Pharmacist participation on physician rounds and adverse drug events in the intensive care unit. JAMA 1999;282:267-270.

-70 Kaushal R, Bates DW, Abramson EL, et al: Unit-based clinical pharmacists' prevention of serious medication errors in pediatric inpatients. Am J Health Syst Pharm 2008;65:12541260.

-71 McDonald CJ: Protocol-based computer reminders, the quality of care and the non-perfectability of man. N Engl J Med 1976;295 1351-1355.

72 Morris AH: Rational use of computerized protocols in the intensive care unit. Crit Care 2001;5:249-254.

-73 Morris A: Treatment algorithms and protocolized care. Curr Opin Crit Care 2003;9:236240.

74 Bates DW, Cohen M, Leape LL: Reducing the frequency of errors in medicine using information technology. J Am Med Inform Assoc 2001;8:299-308.

75 Schiff GD, Leape LL: Commentary: how can we make diagnosis safer? Acad Med 2012;2 135-138.
Bates DW: Preventing medication errors: a summary. Am J Health Syst Pharm 2007; 64(suppl 9):S3-S9;quiz S24-S26.

77 McDonald R, Waring J, Harrison S: Rules, safety and the narrativisation of identity: a hospital operating theatre case study. Sociol Health Illn 2006;28:178-202.

78 Storey J, Buchanan D: Healthcare governance and organizational barriers to learning from mistakes. J Health Organ Manag 2008;22: 642-651.

79 Localio AR, Lawthers AG, Brennan TA, et al: Relation between malpractice claims and adverse events due to negligence. Results of the Harvard Medical Practice Study III. N Engl J Med 1991;325:245-251.

80 Towse A, Danzon P: Medical negligence and the NHS: an economic analysis. Health Econ 1999;2:93-101.

81 National Health Service Litigation Authority. Key facts about our work. www.nhsla.com/ home.htm (accessed October 19, 2012).

82 Dyer C: GPs face escalating litigation. BMJ 1999;7187:830.

83 Fenn P, Diacon S, Gray A, et al: Current cost of medical negligence in NHS hospitals: analysis of claims database. BMJ 2000;320:15671571.

84 Al-Saeed AH: Medical liability litigation in Saudi Arabia. Saudi J Anaesth 2010;4:122126.

85 Rothschild JM, Federico FA, Gandhi TK, et al: Analysis of medication-related malpractice claims: causes, preventability, and costs. Arch Intern Med 2002;162:2414-2420.

86 Kelly WN: Potential risks and prevention. Part 4. Reports of significant adverse drug events. Am J Health Syst Pharm 2001;58: 1406-1412.

87 Nash JJ, Nash AG, Leach ME, et al: Medical malpractice and corticosteroid use. Otolaryngol Head Neck Surg 2011;144:10-15.

88 Abadin SS, Kaplan EL, Angelos P: Malpractice litigation after thyroid surgery: the role of recurrent laryngeal nerve injuries, 19892009. Surgery 2010;148:718-722;disc 722 723.

89 Singer MC, Iverson KC, Terris DJ: Thyroidectomy-related malpractice claims. Otolaryngol Head Neck Surg 2012;146:358-361.

90 Benson JS, Coogan CL: Urological malpractice: analysis of indemnity and claim data from 1985 to 2007. J Urol 2010;184:10861090;quiz 1235.

-91 Badger WJ, Moran ME, Abraham C, et al: Missed diagnoses by urologists resulting in malpractice payment. J Urol 2007;178:25372539.

92 Cottam D, Lord J, Dallal RM, et al: Medicolegal analysis of 100 malpractice claims against bariatric surgeons. Surg Obes Relat Dis 2007; 3:60-66; disc 66-67.

93 Dawson DE, Kraus EM: Medical malpractice and rhinology. Am J Rhinol 2007;21:584-590.

-94 Lydiatt DD, Sewell RK: Medical malpractice and sinonasal disease. Otolaryngol Head Neck Surg 2008;139:677-681.
-95 Mathew R, Asimacopoulos E, Valentine P: Toward safer practice in otology: a report on 15 years of clinical negligence claims. Laryngoscope 2011;121:2214-2219.

$\$ 96$ McGrory BJ, Bal BS, York S, et al: Surgeon demographics and medical malpractice in adult reconstruction. Clin Orthop Relat Res 2009;467:358-366.

97 Upadhyay A, York S, Macaulay W, et al Medical malpractice in hip and knee arthroplasty. J Arthroplasty 2007;22(suppl 2):2-7.

98 McGwin G, Wilson SL, Bailes J, et al: Malpractice risk: trauma care versus other surgical and medical specialties. J Trauma 2008; 64:607-612; disc 612-613.

-99 Ali N: A decade of clinical negligence in ophthalmology. BMC Ophthalmol 2007;7:20.

100 Kiani M, Sheikhazadi A: A five-year survey for dental malpractice claims in Tehran, Iran. J Forensic Leg Med 2009;16:76-82.

101 Chervenak JL: Overview of professional liability. Clin Perinatol 2007;34:227-232.

102 Greenwood C, Newman S, Impey L, et al: Cerebral palsy and clinical negligence litigation: a cohort study. BJOG 2003;110:6-11.

103 Berglund S, Grunewald C, Pettersson H, et al: Severe asphyxia due to delivery-related malpractice in Sweden 1990-2005. BJOG 2008;115:316-323.

104 Uesugi N, Yamanaka M, Suzuki T, et al: Analysis of birth-related medical malpractice litigation cases in Japan: review and discussion towards implementation of a nofault compensation system. J Obstet Gynaecol Res 2010;36:717-725.

105 Fileni A, Magnavita N: A 12-year follow-up study of malpractice claims against radiologists in Italy. Radiol Med 2006;111:10091022.

106 Fileni A, Magnavita N, Mirk P, et al: Radiologic malpractice litigation risk in Italy: an observational study over a 14-year period. AJR Am J Roentgenol 2010;194:1040-1046.

107 Fileni A, Magnavita N, Mammi F, et al: Malpractice stress syndrome in radiologists and radiotherapists: perceived causes and consequences. Radiol Med 2007;112:1069-1084.

108 Hafström L, Johansson H, Ahlberg J: Diagnostic delay of breast cancer - an analysis of claims to Swedish board of malpractice (LÖF). Breast 2011;20:539-542.

109 Hiyama T, Tanaka S, Yoshihara M, et al: Medical malpractice litigation related to gastrointestinal endoscopy in Japan: a two-decade review of civil court cases. World J Gastroenterol 2006;12:6857-6860.

110 Kornstein MJ, Byrne SP: The medicolegal aspect of error in pathology: a search of jury verdicts and settlements. Arch Pathol Lab Med 2007;131:615-618.

111 Hagihara A, Hamasaki T, Abe T: Association between physician explanatory behaviors and substandard care in adjudicated cases in Japan. Int J Gen Med 2011;4:289_ 297. 
112 Hamasaki T, Takehara T, Hagihara A: Physicians' communication skills with patients and legal liability in decided medical malpractice litigation cases in Japan. BMC Fam Pract 2008;9:43.

113 Hamasaki T, Hagihara A: Physicians' explanatory behaviours and legal liability in decided medical malpractice litigation cases in Japan. BMC Med Ethics 2011;12:7.

114 Studdert DM, Mello MM, Gawande AA, et al: Claims, errors, and compensation payments in medical malpractice litigation. N Engl J Med 2006;354:2024-2033.

115 Huycke LI, Huycke MM: Characteristics of potential plaintiffs in malpractice litigation. Ann Intern Med 1994;120:792-798.

116 Ferner RE: Medication errors that have led to manslaughter charges. BMJ 2000;321: 1212-1216.
117 Holbrook J: The criminalization of fatal medical mistakes. BMJ 2003;7424:11181119.

118 Kay N, Green A, McDowell SE, et al: Should doctors who make clinical errors be charged with manslaughter? A survey of medical professionals and members of the public. Med Sci Law 2008;48:317-324

119 Traina F: Medical malpractice: the experience in Italy. Clin Orthop Relat Res 2009; 467:434-442.

120 Lin PJ: Criminal judgments to medical malpractice in Taiwan. Leg Med (Tokyo) 2009; 11(suppl 1):S376-S378.

121 Mello MM, Brennan TA: Deterrence of medical errors: theory and evidence for malpractice reform. Tex L Rev 2001;80:1595.

122 Carroll AE, Parikh PD, Buddenbaum JL: The impact of defense expenses in medical malpractice claims. J Law Med Ethics 2012; 40:135-142.
123 Catino M, Celotti S: The problem of defensive medicine: two Italian surveys. Stud Health Technol Inform 2009;148:206-221.

124 Miller RA, Sampson NR, Flynn JM: The prevalence of defensive orthopaedic imaging: a prospective practice audit in Pennsylvania. J Bone Joint Surg Am 2012;94:e18.

125 Hermer LD, Brody H: Defensive medicine, cost containment, and reform. J Gen Intern Med 2010;25:470-473.

126 Chen XY: Defensive medicine or economically motivated corruption? A Confucian reflection on physician care in China today. J Med Philos 2007;32:635-648.

127 Davies J: Clinical guidelines as a tool for legal liability. An international perspective. Med Law 2009;28:603-613.

28 Hurwitz B: How does evidence based guidance influence determinations of medical negligence? BMJ 2004;329:1024-1028. 